\title{
Prevention of intravascular catheter infection Philippe Eggimann
}

\begin{abstract}
Purpose of review
To review recent evidence supporting the guidelines for preventing catheter-related and catheter-associated infections.

Recent findings

A series of studies has confirmed, over the past few years, that education-based preventive programmes can reduce these infections by one half to two thirds. The evidence supporting some specific measures has increased for the optimal timing for set replacement, for catheter-site dressing with chlorhexidine-impregnated devices, and for the use of some coated or impregnated intravascular devices.

\section{Summary}

Catheter-related and associated infections are largely preventable and should not be viewed as an unaffordable tribute to technical medicine. Improvements in existing techniques and new technologies should all be integrated into a structured process of continuous improvement in the quality of care.
\end{abstract}

\section{Keywords}

catheter, catheter-related infection, infection control, nosocomial infection, prevention

Curr Opin Infect Dis 20:360-369. (c) 2007 Lippincott Williams \& Wilkins.

Department of Adult Intensive Care Medicine and Burn Centre, Centre Hospitalier Universitaire Vaudois (CHUV), Lausanne, Switzerland

Correspondence to Philippe Eggimann, MD, Department of Adult Intensive Care Medicine and Burn Centre, Centre Hospitalier Universitaire Vaudois (CHUV),

$\mathrm{BH}$ 08-609, Bugnon 46, CH-1011 Lausanne, Switzerland

Tel: +41 21314 2923; fax: +4121314 3045;

e-mail: philippe.eggimann@chuv.ch

Current Opinion in Infectious Diseases 2007, 20:360-369

\section{Abbreviations}

CDC Centers for Disease Control and Prevention

CHSS chlorhexidine-silver sulfadiazine

CVC central venous catheter

ICU intensive care unit

(C) 2007 Lippincott Williams \& Wilkins $0951-7375$

\section{Introduction}

Infections associated with the use of intravascular catheters or devices represent $10-20 \%$ of all nosocomial infections. They may complicate the stays of up to $10 \%$ of intensive care unit (ICU) patients. Almost all patients staying in an ICU require at least one intravascular device for fluid/drug administration and approximately half are central venous catheters (CVCs) [1]. According to data from the National Nosocomial Infections Surveillance system, it is estimated that at least $48600 \mathrm{ICU}$ patients develop a CVC-related bloodstream infection every year in US ICUs (approximately five episodes per 1000 catheter-days). These infections, mostly caused by coagulase-negative staphylococci, Staphylococcus aureus, Enterococcus species and Candida species, are associated with considerable morbidity (prolonged length of stay and increased costs) and mortality [ $\left.2^{\bullet}\right]$. Although debated by experts with regard to magnitude, the attributable mortality of these infections may correspond to 500015000 deaths directly caused by catheter-related infections; the benchmarking of rates is currently included in the assessment of quality of care in many institutions $\left[3^{\circ}\right]$.

As for other nosocomial infections, the cornerstone of prevention of intravascular device-associated or related infections relies first on a strict observation of the basic rules of hygiene, of which hand hygiene remains the first and most important procedure [4]. More specific measures, including the use of maximal sterile barriers for insertion, the optimal insertion site, skin preparation, detailed guidelines for catheter care and replacement, and defining particular situations in which the use of antiseptic/antibiotic-coated devices may be used, have been addressed in many clinical studies, and are extensively discussed in regularly updated evidence-based guidelines and recommendations [5-7]. A series of studies has confirmed, over the past few years, that education-based preventive programmes can reduce the incidence of these infections by more than two thirds.

Accordingly, infections associated with or related to intravascular devices are now considered among the most preventable nosocomial infections, and have become a focus for the 100000 Live Campaign of the Institute for Healthcare Improvement $\left[8^{\bullet}\right]$.

\section{Prevention of vascular access-associated and related infections}

Recommendations and guidelines for the prevention of catheter-associated and related infections consisted for 
decades of exhaustive catalogues of many specific measures previously demonstrated to be efficient [5]. Specific measures include the use of maximal sterile barriers during insertion, optimal insertion site preparation, detailed guidelines for catheter insertion and replacement, and defining particular situations in which the use of antiseptic/antibiotic-coated devices may be useful. Data from surveillance programmes repetitively showed that they are generally not used or are insufficiently applied.

As with any nosocomial infections, however, their prevention should rely first on general measures, including on a strict observation of the basic rules of hygiene, of which hand hygiene represent the first and most important procedure [4]. They should be continuously emphasized and reinforced before considering any specific measures [9].

Early in the 2000s four cohort studies showed that multimodal educational programmes were able to reduce dramatically the rate of catheter-associated and related infections by more than two thirds [10-13]. These programmes all consisted of the systematic education of the personnel in charge of the insertion and care of intravascular access in ICUs. Emphasizing general measures first, including a strict observation of the basic rules of hygiene, these interventions produced a standardization of care at the bedside. Elaborated by personnel on the ward in close collaboration with infection control specialists, and implemented using communication and education tools, active participation and positive feedback, they all resulted in important behavioural changes.

The concept of preventing catheter-related infections has evolved accordingly, and education-based programmes were recommended as a first-line target in the 2002 renewed guidelines for the prevention of intravascular device-related infections [7].

The objective of this paper is to review further important evidence on a series of topics that has appeared in the literature since the publication of these guidelines.

\section{Education-based programmes}

Increasing amounts of evidence come from at least 17 additional cohort studies, confirming that when included in education-based programmes, combined specific measures can reduce intravascular device-associated and related infections by one half to two thirds (Table 1) $\left[14-25,26^{\bullet \bullet}-30^{\bullet \bullet}\right]$.

In Table 1 the impact of multimodal educational programmes that target the prevention of intravascular device-related infections in adult intensive care units, between 2000-2006 is discussed. The interventions are catergorized as follows:

Sherertz et al. [10]: Education consisted of one day course including 1-h stations in which participants received training in: (1) blood draws through vascular lines; (2) arterial puncture for obtaining an arterial blood gas; (3) insertion of arterial catheters and central venous catheters (CVCs); (4) urinary catheter insertion; (5) lumbar puncture; (6) peripheral venous catheter insertion; (7) phlebotomy.

Eggimann et al. [11]: Multiple-approach intervention targeted at the reduction of vascular-access infections including a 30-min slide show and practical demonstrations completed by individual in-service training. The programme included detailed written information on clinical pathways on hand hygiene procedures, for vascular access insertion, and device maintenance and use.

Yoo et al. [12]: The intervention included: (1) distribution of new guidelines for the care of catheters (skin and hand disinfection, occlusive gauze dressing) with insertion sets that included a sterile gown and gloves, a mask, and a large sterile drape; (2) daily surveillance in the ICU to monitor all catheters by a checklist; (3) recommendation for systematic blood cultures and removal of the catheter if the patient develops fever of unknown origin or any symptoms or signs of CR-BSI.

Coopersmith et al. [13]: Based on audits to determined differences between the Centers for Disease Control and Prevention (CDC) guidelines and current practice, a task force built an education intervention to improve practices related to CVC insertion and care: (1) 10-Page self-study module with pre and post-question exam including the following topics: (a) epidemiology and scope of the problem; (b) risk factors; (c) aetiology; (d) definition; and (e) methods to decrease risk. Specific risk-reduction strategies addressed included: (i) handwashing and aseptic techniques; (ii) methods for detecting potential clinical signs and symptoms of local infection; (iii) techniques for sending catheter-tip culture; (iv) routine catheter site care; $(\mathrm{v})$ replacing administration sets and fluids; (vi) cleaning and changing injection ports and luer-lock caps; (vii) how to handle parenteral fluids and multidose vials; and (viii) procedure for drawing blood cultures. (2) Guidelines for catheter maintenance included changing injection caps and intravenous tubing for fluids and medications every $72 \mathrm{~h}$ (or immediately if blood accumulated in or around the cap or its integrity was compromised). In addition, transparent line dressings were replaced every 7 days, whereas gauze dressings (which were used solely when there was bleeding or oozing at the insertion site) were changed every $48 \mathrm{~h}$. The guidelines also recommended that dressings that 


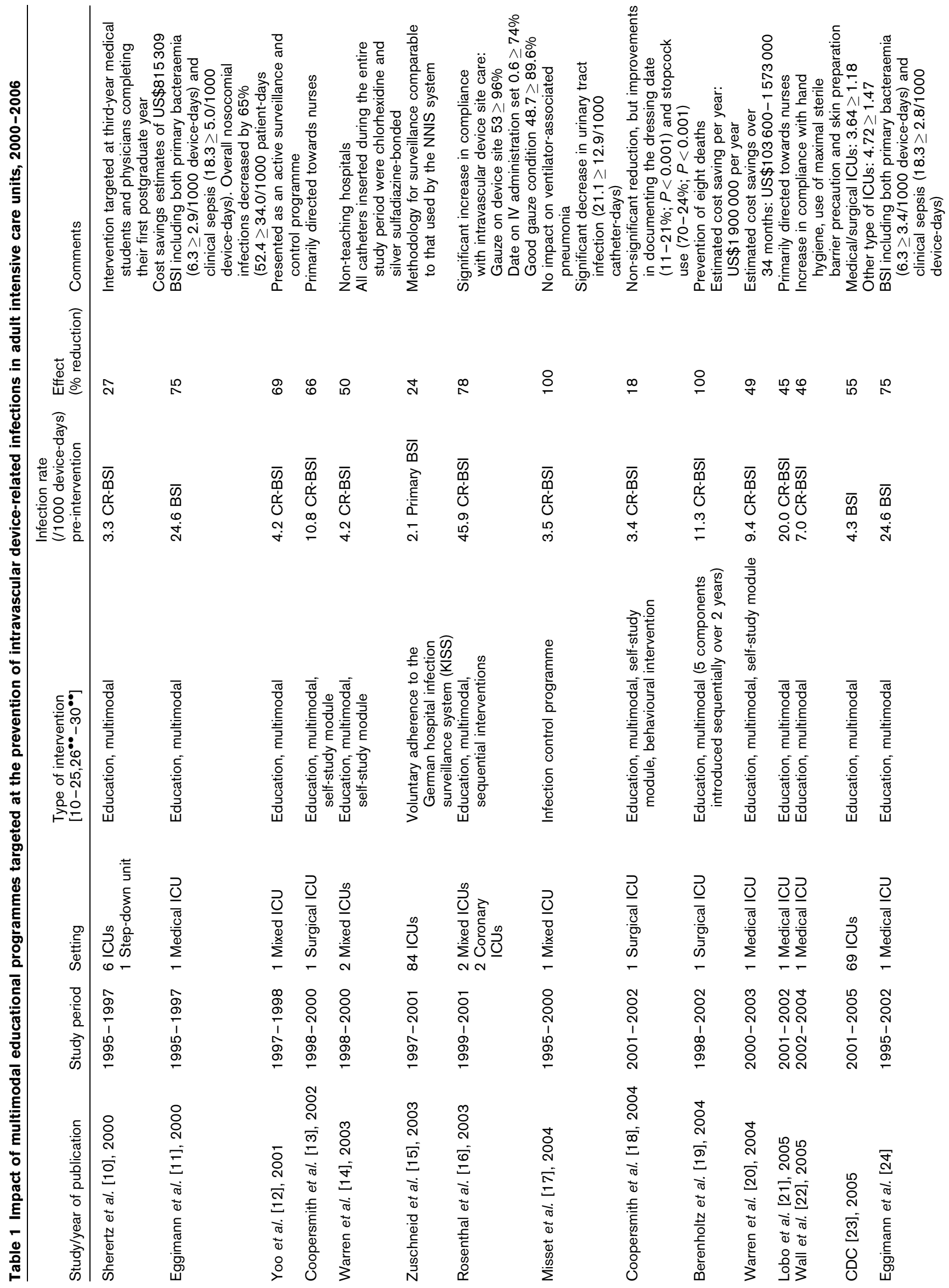




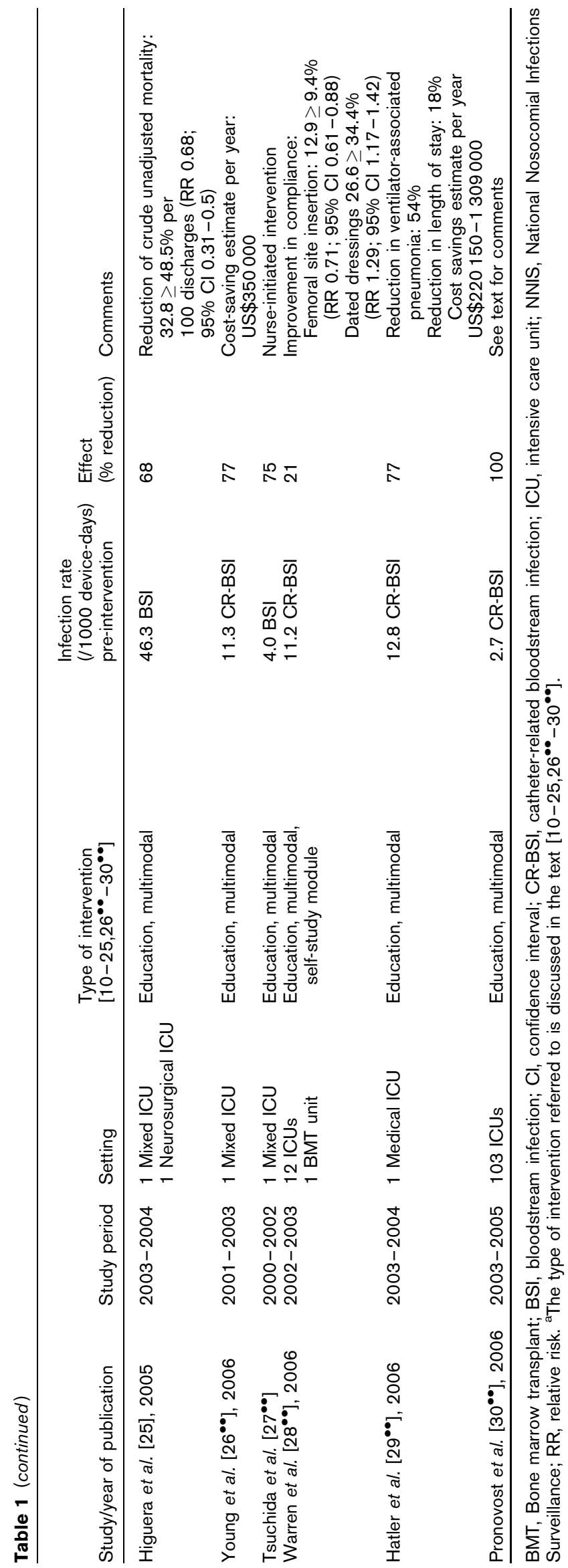

were either soiled or no longer occlusive be replaced immediately.

Warren et al. [14]: Identical to the interventions in Coopersmith et al. [13].

Zuschneid et al. [15]: The Hospital Infection Surveillance System (Krankenhaus Infektions Surveillance System; KISS) uses CDC definitions for reporting in ICUs and focuses on primary bloodstream infections, urinary tract infections, pneumonia, and bronchitis. The participating hospitals receive their rates of infection and device use twice yearly so as to provide feedback and encourage infection control activities in the participating ICUs.

Rosenthal et al. [16]: The intervention included three steps: (1) active surveillance for intravascular deviceassociated infections and compliance with intravascular device site care; (2) education and training of all healthcare workers for catheter care on the basis of the CDC guidelines; (3) monthly performance feedback documenting rates of compliance with handwashing, gauze on CVC insertion sites, dates on intravenous administration sets, and maintaining the condition of catheter gauze dressings.

Misset et al. [17]: Implementation of infection control practices produced by the ICU staff together with the infection control unit according to the CDC recommendations including general measures such as hand hygiene and antibiotic control, and specific guidelines targeted at the prevention of ventilator-associated pneumonia and of intravascular device-related infections.

Coopersmith et al. [18]: Identical to Coopersmith 2002 [13] with an additional multifactorial behavioural intervention targeted at improving compliance deficiencies noted in audits in intravascular device insertion and maintenance.

Berenholtz et al. [19]: Five components sequentially introduced from February 1999 to November 2001: (1) educational intervention to increase provider awareness of evidence-based infection control practices; (2) creation of a central catheter insertion cart; (3) asking providers daily whether catheters can be removed; (4) implementation of a checklist to be completed by the bedside nurse; (5) empowerment of nurses to stop procedures if guidelines are not followed.

Warren et al. [20]: The intervention included: (1) review and update of hospital or unit policies and procedures concerning the insertion and the use of devices by infection control team and ICU staff and members; (2) staff education by three methods: (a) didactic lectures and presentations; (b) self-study module with pre and posttest; (c) fact sheets and posters; (3) the primary messages 
were: (i) hand hygiene procedures; (ii) avoidance of femoral site; (iii) maximal sterile barrier precautions for insertion; (iv) precise guidelines for catheter handling and dressing.

Lobo et al. [21]: The intervention consisted of: (1) observation and 10 pretest questions about hand hygiene and catheter insertion and handling; (2) education classes and poster for hand hygiene and catheter insertion (skin preparation) and handling (dressing replacement).

Wall et al. [22]: The intervention consisted of: (1) education: provider, trainee supervision, insertion site, hand hygiene procedures, skin antisepsis, and use of maximal sterile barriers; (2) standardized checklist for nurse including: hand hygiene, trainee supervision, maximal sterile barriers, skin preparation with chlorhexidine (2\% solution), circumstance of catheter insertion; (3) audits and monthly feedback.

CDC [23]: The intervention consisted of: (1) education for catheter insertion practices (maximum sterile barrier precautions; chlorhexidine solution for skin disinfection; avoidance of the femoral insertion site; guidelines for dressing; removal of catheters no longer indicated); (2) promotion of educational module on device-associated bloodstream infections and strategies for their prevention; (3) promotion of standardized tools for recording adherence to recommendations; (4) promotion of a standardized list of contents for catheter insertion kits; (5) measurement of CVC-associated bloodstream infection rates, with quarterly reports allowing anonymous benchmarking between participating ICUs.

Eggimann et al. [24]: Identical to the interventions in Eggimann et al. [11].

Higuera et al. [25]: The intervention consisted of: (1) education: $1 \mathrm{~h}$ classes on epidemiology of nosocomial infections, on hand hygiene, disinfection, on prevention of intravascular device-associated bloodstream infections, on prevention of ventilator-associated pneumonia, and on prevention of catheter-associated urinary tract infections; (2) performance feedback: monthly chart (hand hygiene compliance, gauze on CVC insertion sites, dates on intravenous administration sets, maintenance of gauze dressings on catheter sites) posted on the walls of the ICUs; (3) the infection control guidelines included the introduction of the use alcohol hand-rubs or hand washing with povidone-iodine soap.

Young et al. $\left[26^{\bullet \bullet}\right.$ ]: The intervention consisted of: (1) educational sessions that emphasized the use of maximal sterile barrier precautions and the use of chlorhexidine for skin antisepsis during insertion of CVCs; (2) introduction of a special customized CVC kit including a large sterile drape ( $41^{\prime \prime}$ by $55^{\prime \prime}$ with $4^{\prime \prime}$ fenestration) and $2 \%$ chlorhexidine gluconate in $70 \%$ isopropyl alcohol for skin antisepsis.

Tsuchida et al. [27 $7^{\bullet \bullet}$ ]: The intervention consisted of: (1) enhanced skin preparation by scrubbing with regular bathing soap and tap water; (2) a new method for stabilization of the catheter inserted into the internal jugular vein, in which an additional dressing was placed over the sterilized dressing; (3) education of the staff for maximal sterile precautions by teaching staff members and displaying posters; (4) use of a check-list and observation of catheter insertion by link nurses to monitor compliance; (5) selection of a disinfectant that requires shorter contact time and has longer residual effect (from 10\% povidoneiodine to $0.5 \%$ chlorhexidine- $78 \%$ ethanol).

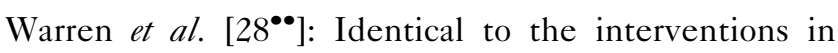
Warren et al. [20].

Hatler et al. [29 $9^{\bullet \bullet}$ : The intervention consisted of: (1) staff members received a one-page document detailing new strategies to address the prevention of ventilatorassociated pneumonia and CR-BSI, thus increasing their abilities to control and perform the desired behaviours; (2) chart of expected activities was posted in each patient's room; (3) daily rounds, led by the unit's nursing supervisor, helped establish adherence to the targeted procedures as 'normal' behaviour for staff members; (4) daily goals were posted on white boards in patients' rooms; (5) regular feedback about the rates; (6) use of a rapid-cycle approach to increase the flexibility of implementing the change measured for 2-4 weeks.

Pronovost et al. [30 ${ }^{\bullet \bullet}$ ]: the intervention included five of the highly ranked (CDC category IA) evidence-based recommendations identified as having the greatest effect on the rate of catheter-related bloodstream infection and the lowest barriers to implementation [7]. The recommended procedures were: (1) hand washing; (2) using full-barrier precautions during the insertion of CVCs; (3) cleaning the skin with chlorhexidine; (4) a subclavian site is preferred and the femoral site is avoided if possible; (5) removing unnecessary catheters. They were promoted by local physicians and nurses team leaders as follows: (a) staff were educated about practices to control infection and harm resulting from catheter-related bloodstream infections; (b) central-line cart with necessary supplies was promoted or created; (c) a checklist was used to ensure adherence to infection-control practices; (d) providers were stopped (in non-emergency situations) if these practices were not being followed; (e) the removal of catheters was discussed at daily rounds; (f) the teams received feedback regarding the number and rates of catheter-related bloodstream infections at monthly and quarterly meetings, respectively. 
The successful prevention of infections has not only been demonstrated in cases of high initial rates, but also in institutions with considerable experience in the prevention of nosocomial infections and initially low rates of infections $\left[10,15,17,18,30^{\bullet \bullet}\right]$. A majority of these programmes has been shown to be largely cost-effective. Moreover, such programmes may also work when impregnated catheters were already in use before the intervention [14].

The most recent report concerned an interventional cohort study involving 103 ICUs in 67 hospitals in Michigan with more than 375000 catheter-days of observation [ $\left[30^{\bullet \bullet}\right.$. The intervention, detailed in Table 1 , was remarkable in several points. The programme was promoted by local physicians and nurses organized by team leaders. It was supported by the introduction of daily goal sheets to improve clinician-to-clinician communication within the ICUs. Furthermore, it was integrated in a comprehensive unit-based safety programme to improve the safety culture, which also included an intervention to reduce the incidence of ventilator-associated pneumonia. Over the 18 months after the implementation of the intervention, the median rate of catheter-related bloodstream infections was reduced by $66 \%$, from 2.7 (mean 7.7) to 0 (mean 1.4) per 1000 catheter-days.

The series of mono and multicentre studies, which includes a large variety of ICUs all around the world, strongly suggests that the process of training is progressively, and hopefully definitely, moving from the traditional widespread approach 'see one, do one, teach one' to an integrated and structured process of continuous improvement of the quality of care.

\section{Optimal timing for intravenous administration set replacement}

The current guidelines recommend, scored with the highest level of evidence, to replace administration sets, including secondary sets and add-on devices, no more frequently than at 72-h intervals, unless a catheterrelated infection is suspected or documented. It is recommended, with a slightly less high level of evidence, to decrease this delay to 24-h intervals for the administration of blood products or lipid emulsions [7].

Gilles et al. [31] from the Cochrane Anesthesia Group performed a systematic review to identify the optimal interval for the routine replacement of intravenous administration sets. From their analysis of the data extracted from 13 out of 23 studies, the authors concluded that administration sets that do not contain lipids, blood or blood products may be left in place for intervals of up to $96 \mathrm{~h}$ without an increase in the risk of infection. There was no evidence to suggest that administration sets containing lipids should not be changed every $24 \mathrm{~h}$ as currently recommended. In addition, subgroup analysis found no differences between CVCs and peripheral catheters, nor between participants who did and did not receive parenteral nutrition, or between children and adults.

\section{Needleless catheter access systems}

Designed to reduce the incidence of sharp injuries to healthcare workers, needleless infusion systems have been developed by several manufacturers. They have not been proved to be able to prevent catheter-related infections, and were scored as an unresolved issue in the 2002 guidelines [7].

Niel-Weisse et al. [32 ${ }^{\bullet \bullet}$ ] recently reviewed five randomized trials evaluating the effect of vascular access needleless closed systems on catheter-related infections among hospitalized patients. The quality of these trials and the way they were reported were generally unsatisfactory, and their heterogeneity did not allow a meta-analysis to be performed. Nevertheless, there was a trend towards the needleless closed devices decreasing catheter-related bloodstream infections. Catheter tip and hub colonization were significantly reduced in one out of three and two out of three studies, respectively. From their detailed review, the authors specified that in contrast to several previous reports, they did not find a trend in needleless closed devices towards an increased risk of infection [33,34]. They concluded that there is currently insufficient evidence to recommend needleless closed vascular devices, and that new studies are necessary to confirm that these systems reduce catheter-related infections.

\section{Catheter site dressing with chlorhexidine-impregnated devices}

Gluconate of chlorhexidine is a powerful antimicrobial agent widely used for surgical scrub and for hand hygiene, with a good safety profile. When used as a skin disinfectant, it is more effective than povidine-iodine solutions in reducing catheter-related bloodstream infections [35]. Devices impregnated with chlorhexidine gluconate (Biopatch; Ethicon Inc., Somerville, New Jersey, USA) are able to release it up to 10 days over the skin surface when placed over the epidural catheter insertion site [36]. Whether these devices could be routinely used in the care of short-term intravascular accesses, however, remained unresolved in the 2002 guidelines [7].

Ho and Litton [ $37^{\bullet \bullet}$ ] found 10 prospective, randomized, controlled clinical trials comparing chlorhexidineimpregnated dressings with placebo or povidine-iodine dressing, and included eight of them in a meta-analysis. The chlorhexidine-impregnated dressing reduced the risk of intravascular catheter or exit-site colonization [14.8 versus $26.9 \%$; odds ratio (OR) 0.47 ; $95 \%$ confidence interval (CI) $0.34-0.65 ; P<0.00001]$. After excluding the study that compared chlorhexidine-impregnated 
dressings with povidine-iodine dressings, they were associated with a significant reduction in catheter-related bloodstream infections when compared with placebo (OR 0.40; 95\% CI $0.21-0.75 ; P=0.004$ ). Local cutaneous reactions to chlorhexidine-impregnated dressings were reported in $5.6 \%$ of patients in three studies (OR 8.17; $95 \%$ CI $1.19-56.14 ; P=0.04$ ), and $96 \%$ of these reactions occurred in neonatal patients. The number needed to prevent one episode of bloodstream infection was 142 for an average period of catheterization of 10 days and a change of dressings every 5 days. The cost of preventing one catheter-related bloodstream infection was estimated to be US\$532.5. The authors concluded that the use of chlorhexidine-impregnated dressings is safe and may be cost-effective in adult patients with intravascular devices. This should now be confirmed in a large prospective, randomized controlled trial.

\section{Coated or impregnated intravascular devices}

The use of antibiotic or antiseptic-impregnated catheters reduces device-related infections but remains controversial. In the last guidelines, experts recommended considering the use of such devices if the rate of catheterrelated bloodstream infections remains higher than 3.3 episodes per 1000 CVC-days, after having enhanced other preventive measures, including multimodal educative programmes [7].

Several meta-analyses that included studies published after the release of those guidelines appeared in the literature.

Walder et al. [38] reviewed the impact of coated devices in 23 trials, including chlorhexidine-silver sulfadiazine (CHSS) internal coating in 12, silver-based coating or cuffing in seven, and various antibiotic coatings in five. The major finding of that meta-analysis was that in five trials on CHSS coating (1269 CVCs), in which the average insertion time ranged from 5.2 to 7.5 days (median 6 days), the risk of bloodstream infections significantly decreased from $4.1 \%$ in controls to $1.9 \%$ with anti-infective catheters (OR 0.48 ; 95\% CI $0.25-$ 0.91). In five additional trials on CHSS coating (1544 GVCs), in which the average insertion time ranged from 7.8 to 20 days (median 12 days), the risk of bloodstream infections was $4.5 \%$ in controls and $4.2 \%$ with anti-infective catheters (OR 0.94; 95\% CI 0.58-1.54). The authors concluded that antibiotic and CHSS coatings are anti-infective for short insertion times (less than one week). For longer insertion times (more than one week), there were no data on antibiotic coating, and some evidence of a lack of effect for CHSS coating.

Geffers et al. [39] assessed the methodological quality of 11 randomized controlled studies (3131 catheters) with
CHSS-impregnated catheters (on their internal surface only) and the effect on catheter-related infection. The pooled data did not show a significant reduction of catheter-related bloodstream infections (OR 0.69; 95\% CI 0.46-1.03). Statistical significance was achieved only when both studies with a more appropriate type of definition were separated out (OR 0.58; 95\% CI 0.46$0.96)$, or when only studies with a short average duration of catheterization (maximum 8 days) were considered (OR 0.34; 95\% CI 0.15-0.75). The authors highlighted the fact that all methods of diagnosing catheter colonization used in the studies were evaluated with uncoated catheters. They suggested that failure to use neutralizers could lead to an underestimation of the rate of catheterrelated bloodstream infections; conversely, the absence of inhibitory agents may lead to an overestimation of the antibacterial efficacy of coated catheters.

Falagas et al. [40 ${ }^{\bullet \bullet}$ ] performed a meta-analysis of eight prospective randomized clinical trials including a total of 1715 rifampicin-based impregnated CVCs, 956 uncoated catheters, 451 catheters coated on their internal surface by CHSS, and 237 silver-platinum-carbon-impregnated catheters. Overall, the rifampicin/minocycline-impregnated catheters significantly reduced bacterial colonization (OR 0.46; 95\% CI 0.31-0.69) and catheter-related bloodstream infections (OR 0.23 ; 95\% CI 0.14-0.40). On the basis of the available data, no clear conclusions could be drawn regarding the impact of the use of rifampicin/ minocycline-impregnated CVCs on the development of antimicrobial resistance. The authors concluded that these catheters are safe and effective in reducing the rate of catheter colonization and related infections. Regarding the development of resistance, reviewing the effectiveness and safety of rifampicin-based regimens in eradicating $S$. aureus carriage, the same authors recently concluded that the available evidence suggested that oral rifampicin is effective in its eradication $\left[41^{\circ}\right]$. They also specified that the development of resistance occurs in a considerable proportion of patients.

The potential greater efficacy of catheters coated with minocycline/rifampicin compared with those coated with CHSS in preventing infections has largely been attributed to the fact that CHSS coating concerned only the internal lumen of the device [42]. Since then, a second generation of CHSS catheters with coating of both the internal and the external surface was produced by the manufacturer. At least three studies [43-45], including two large multicentre ICU studies, have failed to demonstrate a significant reduction in catheter-related bloodstream infections. All showed a significant reduction in bacterial colonization. The overall low rate of infection in the placebo arms could, however, argue in favour of insufficient power. By pooling the data, Gastmeier and Geffers [46] demonstrated a significant reduction in the 
rate of catheter-related bloodstream infections [relative risk (RR) 0.40; 95\% CI 0.18-0.90].

New composite catheters, consisting of polyurethane combined with silver, carbon, and platinum, have been introduced onto the market. They were demonstrated to be able to release ions of silver with prolonged antimicrobial activity (oligon) [47-51]. Those studies showed a significant reduction in bacterial colonization. Even by pooling the data (822 impregnated versus 833 non-impregnated catheters), Gastmeier and Geffers [46] did not find a significant reduction in the rate of catheterrelated bloodstream infections (RR 0.81; 0.46-1.45).

To summarize the information of the many randomized studies, the impact of these devices is in the same range as those of the educational programmes. Nevertheless, they should never replace them. It should be highlighted that in at least one of the studies demonstrating a significant reduction in infections after the introduction of a multimodule educational programme, coated catheters were used during the entire study period [14].

Borschel et al. $\left[52^{\bullet \bullet}\right.$ ] recently reported the impact in a real-world setting after the introduction of CHSS CVCs (second generation with internal and external coating) in all consecutive patients requiring central venous access in six adult ICUs at a large, tertiary care teaching hospital. The intervention significantly increased the rate of reduction of the incidence of catheter-related bloodstream infections by approximately $4 \%$ per month, corresponding to an overall $35 \%$ relative risk reduction over a 16 -month period (from approximately nine to less than four episodes per 1000 device-days). With 41 infections avoided for approximately 1647 coated catheters inserted, the authors estimated that the intervention saved US $\$ 266325$ over one year. Assuming that not all of the observed effect was caused by the coated catheters, the intervention would remain cost-effective even if only $13 \%$ of the observed decrease in infection rate was attributable to it. Furthermore, the intervention was associated with a significant reduction in the rate of vancomycin use.

If such a positive and cost-effective impact of antisepticcoated devices in a real-world setting could be confirmed in large multicentre studies, it may contribute to increase the level of evidence to include them in all strategies targeted at the reduction of catheter-related infections.

\section{New areas of development}

Antibiotic-lock or flush solutions are not routinely recommended for the prevention of device-related infections by the 2002 guidelines [7]. They were confirmed to be able to reduce significantly the rate of catheter-related infections and be useful in the treatment of persistent bacteraemia in long-term catheters by two recently pub- lished meta-analyses $\left[53^{\bullet \bullet}, 54^{\bullet}\right]$. These techniques should, however, be restricted to this setting when infection with coagulase-negative staphylococci is documented but without systemic signs of sepsis or secondary dissemination [55]. Accordingly, as a result of the risk of promoting the spread of resistance, and the lack of data in other types of settings, they should continue not to be used routinely.

The 2002 guidelines recommend the use of a $2 \%$ solution of chlorhexidine for skin antisepsis before intravascular device insertion and further maintenance care. The majority of the commercially available chlorhexidinebased solutions for use with intravascular devices consist of $2 \%$ chlorhexidine gluconate in $70 \%$ isopropyl alcohol. Medical-grade ethanol has already been in use for many years for the management or prevention of the occlusion of intravascular devices used for parenteral nutrition [56,57]. A series of reports has suggested that 25-70\% of ethanol used as a lock solution may be of value both as an adjunct to the treatment of intravascular devicerelated bloodstream infections and for the prevention of infection with the use of long-term intravascular devices $\left[58,59^{\bullet}, 60^{\bullet}\right]$. Crnich and colleagues [61] demonstrated that a $70 \%$ ethanol lock solution has a negligible impact on the mechanical properties of polyetherurethane and silicone catheters, despite continuous exposure times as long as 10 weeks. These findings should prompt further studies of ethanol as an antiinfective lock solution for the prevention and treatment of intravascular device-related bloodstream infections in other settings, including shorter catheterization times.

\section{Conclusion}

Catheter-related and associated infections are largely preventable and should not be viewed as an unaffordable tribute to technical medicine. Their prevention is a very active and stimulating field for clinical research. Improvements in many specific technical measures, such as new catheter securement or fixation devices, will probably continue to be studied. New technologies are stimulating the design of new types of impregnated devices. After being shown to be useful, all these improvements should then be integrated into structured multimodal educational programmes. Moreover, these interventions should all be integrated in a structured process of continuous improvement of the quality of care.

\section{References and recommended reading}

Papers of particular interest, published within the annual period of review, have been highlighted as:

- of special interest

- of outstanding interest

Additional references related to this topic can also be found in the Current World Literature section in this issue (p. 436).

1 National Nosocomial Infections Surveillance (NNIS). System report, data summary from January 1992 through June 2004, issued October 2004. Am J Infect Control 2004; 32:470-485. 
2 Warren DK, Quadir WW, Hollenbeak CS, et al. Attributable cost of catheter- associated bloodstream infections among intensive care patients in a nonteaching hospital. Crit Care Med 2006; 34:2084-2089.

After adjusting for potential confounders in a cohort of 41 catheter-associated bloodstream infections, these infections resulted in an attributable cost of US\$11971, ICU length of stay of 2.41 days, and hospital length of stay of 7.54 days.

3 Wenzel RP, Edmond MB. Team-based prevention of catheter-related infec- $\quad$ tions. N Engl J Med 2006; 355:2781-2783.

In a well-documented editorial, after new estimations of the epidemiology of catheter-related infection in the United States, the authors recommended to the medical community to adhere to such concepts of caring behaviour that consistently lead to safety and comfort for patients.

4 Boyce JM, Pittet D. Guideline for hand hygiene in health-care settings. Recommendations of the Healthcare Infection Control Practices Advisory Committee and the HICPAC/SHEA/APIC/IDSA Hand Hygiene Task Force. Society for Healthcare Epidemiology of America/Association for Professionals in Infection Control/Infectious Diseases Society of America. MMWR Morb Mortal Wkly Rep 2002; 51:1-45.

5 Pearson ML. Guideline for prevention of intravascular device-related infections. Hospital Infection Control Practices Advisory Committee. Infect Control Hosp Epidemiol 1996; 17:438-473.

6 Mermel LA, Farr BM, Sherertz RJ, et al. Guidelines for the management of intravascular catheter-related infections. Clin Infect Dis 2001; 32:12491272.

7 O'Grady NP, Alexander M, Dellinger EP, et al. Guidelines for the prevention of intravascular catheter-related infections. Centers for Disease Control and Prevention. Morb Mortal Wkly Rep 2002; 51:1-29.

8 Berwick DM, Calkins DR, McCannon CJ, Hackbarth AD. The 100000 lives

- campaign: setting a goal and a deadline for improving healthcare quality. JAMA 2006; 295:324-327.

Presentation of the 100000 Lives Campaign (Institute for Healthcare Improvement) with a summary of the six bundles retained, including those for preventing central-line infections. This bundle highlights five evidence-based measures (extracted from the 2002 guidelines) that appear to be particularly important in reducing the risk of central-line infection, and a daily review of line necessity, with prompt removal of unnecessary lines.

9 Pittet D, Hugonnet S, Harbarth S, et al. Effectiveness of a hospital-wide programme to improve compliance with hand hygiene. Lancet $2000 ; 356$ $1307-1312$.

10 Sherertz RJ, Ely EW, Westbrook DM, et al. Education of physicians-in-training can decrease the risk for vascular catheter infection. Ann Intern Med 2000, 132:641-648.

11 Eggimann $\mathrm{P}$, Harbarth S, Constantin MN, et al. Impact of a prevention strategy targeted at vascular-access care on incidence of infections acquired in intensive care. Lancet 2000; 355:1864-1868.

12 Yoo S, Ha M, Choi D, Pai H. Effectiveness of surveillance of central catheterrelated bloodstream infection in an ICU in Korea. Infect Control Hosp Epidemiol 2001; 22:433-436.

13 Coopersmith CM, Rebmann TL, Zack JE, et al. Effect of an education program on decreasing catheter-related bloodstream infections in the surgical intensive care unit. Crit Care Med 2002; 30:59-64.

14 Warren DK, Zack JE, Cox MJ, et al. An educational intervention to prevent catheter-associated bloodstream infections in a nonteaching, community medical center. Crit Care Med 2003; 31:1959-1963.

15 Zuschneid I, Schwab F, Geffers C, et al. Reducing central venous catheterassociated primary bloodstream infections in intensive care units is possible: data from the German nosocomial infection surveillance system. Infect Control Hosp Epidemiol 2003; 24:501-505.

16 Rosenthal VD, Guzman S, Pezzotto SM, Crnich CJ. Effect of an infection control program using education and performance feedback on rates of intravascular device-associated bloodstream infections in intensive care units in Argentina. Am J Infect Control 2003; 31:405-409.

17 Misset B, Timsit JF, Dumay MF, et al. A continuous quality-improvement program reduces nosocomial infection rates in the ICU. Intensive Care Med $2004 ; 30: 395-400$.

18 Coopersmith CM, Zack JE, Ward MR, et al. The impact of bedside behavior on catheter-related bacteremia in the intensive care unit. Arch Surg 2004; 139 131-136.

19 Berenholtz SM, Pronovost PJ, Lipsett PA, et al. Eliminating catheter-related bloodstream infections in the intensive care unit. Crit Care Med 2004; 32: 2014-2020.

20 Warren DK, Zack JE, Mayfield JL, et al. The effect of an education program on the incidence of central venous catheter-associated bloodstream infection in a medical ICU. Chest 2004; 126:1612-1618.
21 Lobo RD, Levin AS, Gomes LM, et al. Impact of an educational program and policy changes on decreasing catheter-associated bloodstream infections in a medical intensive care unit in Brazil. Am J Infect Control 2005; 33:83-87.

22 Wall RJ, Ely EW, Elasy TA, et al. Using real time process measurements to reduce catheter related bloodstream infections in the intensive care unit. Qual Safety Healthcare 2005; 14:295-302.

23 Centers for Disease Control and Prevention. Reduction in central lineassociated bloodstream infections among patients in intensive care units Pennsylvania, April 2001-March 2005. MMWR Morb Mortal Wkly Rep $2005 ; 54: 1013-1016$.

24 Eggimann $\mathrm{P}$, Hugonnet $\mathrm{S}$, Sax $\mathrm{H}$, et al. Long-term reduction of vascular access-associated bloodstream infection. Ann Intern Med 2005; 142:875876.

25 Higuera F, Rosenthal VD, Duarte $\mathrm{P}$, et al. The effect of process control on the incidence of central venous catheter-associated bloodstream infections and mortality in intensive care units in Mexico. Crit Care Med 2005; 33: 2022-2027.

26 Young EM, Commiskey ML, Wilson SJ. Translating evidence into

- practice to prevent central venous catheter-associated bloodstream infections: a systems-based intervention. Am J Infect Control 2006; 34:503-506

An infection control intervention designed to facilitate the use of maximal sterile barrier precautions and the use of chlorhexidine for skin antisepsis during the insertion of CVCs. See Table 1 for summary.

27 Tsuchida T, Makimoto K, Toki M, et al. The effectiveness of a nurse-initiated

-• intervention to reduce catheter-associated bloodstream infections in an urban acute hospital: an intervention study with before and after comparison. Int J Nurs Stud 2006; 23 December [Epub ahead of print].

An infection nurse-initiated multimodal intervention designed to reduce catheterrelated bloodstream infections. See Table 1 for summary.

28 Warren DK, Cosgrove SE, Diekema DJ, et al. A multicenter intervention to - prevent catheter-associated bloodstream infections. Infect Control Hosp Epidemiol 2006; 27:662-669.

An educational multicentre intervention to prevent catheter-associated bloodstream infections. See Table 1 for summary.

29 Hatler CW, Mast D, Corderella J, et al. Using evidence and process improve-

- ment strategies to enhance healthcare outcomes for the critically ill: a pilot project. Am J Crit Care 2006; 15:549-555.

A multidisciplinary, environmentally tailored approach to prevent ventilatorassociated pneumonia and catheter-associated bloodstream infections in a medical ICU. See Table 1 for summary.

30 Pronovost $\mathrm{P}$, Needham D, Berenholtz $\mathrm{S}$, et al. An intervention to decrease

- catheter-related bloodstream infections in the ICU. N Engl J Med 2006; 355:2725-2732.

An educational multicentre intervention to prevent catheter-associated bloodstream infections and ventilator-associated pneumonia. See Table 1 and text for summary and comments.

31 Gillies D, O'Riordan L, Wallen $M$, et al. Optimal timing for intravenous administration set replacement. Cochrane Database Syst Rev 2005; 19: CD003588.

32 Niel-Weise BS, Daha TJ, Van den Broek PJ. Is there evidence for recommend - ing needleless closed catheter access systems in guidelines? A systematic review of randomized controlled trials. J Hosp Infect 2006; 62:406-413.

A systematic review of five randomized trials evaluating the effect of vascular access needleless closed systems on catheter-related infections among hospitalized patients. See text for summary and comments.

33 Danzig LE, Short $\sqcup$, Collins $\mathrm{K}$, et al. Bloodstream infections associated with a needleless intravenous infusion system in patients receiving home infusion therapy. JAMA 1995; 273:1862-1864.

34 McDonald LC, Banerjee SN, Jarvis WR. Line-associated bloodstream infections in pediatric intensive-care-unit patients associated with a needleless device and intermittent intravenous therapy. Infect Control Hosp Epidemiol 1998; 19:772-777.

35 Chaiyakunapruk N, Veenstra DL, Lipsky BA, Saint S. Chlorhexidine compared with povidone-iodine solution for vascular catheter-site care: a meta-analysis. Ann Intern Med 2002; 136:792-801.

36 Shapiro JM, Bond EL, Garman JK. Use of a chlorhexidine dressing to reduce microbial colonization of epidural catheters. Anesthesiology 1990; 73:625631.

37 Ho KM, Litton E. Use of chlorhexidine-impregnated dressing to prevent - vascular and epidural catheter colonization and infection: a meta-analysis. J Antimicrob Chemother 2006; 58:281-287.

A systematic review of 10 prospective randomized controlled clinical trials comparing chlorhexidine-impregnated dressings with placebo or povidine-iodine dressings. See text for summary and comments. 
38 Walder B, Pittet D, Tramer M. Benefit of antiseptic and antimicrobial coating of central venous catheters: a systematic review. Infect Control Hosp Epidemiol 2002; 23:748-756.

39 Geffers C, Zuschneid I, Eckmanns T, et al. The relationship between methodological trial quality and the effects of impregnated central venous catheters. Intensive Care Med 2003; 29:403-409.

40 Falagas ME, Fragoulis K, Bliziotis IA, Chatzinikolaou I. Rifampicin-impregnated

- central venous catheters: a meta-analysis of randomized controlled trials. J Antimicrob Chemother 2007; 59:359-369.

A meta-analysis of eight prospective randomized clinical trials comparing rifampicin- based impregnated central venous catheters with uncoated catheters, with catheters coated on their internal surface by CHSS and with silver-platinumcarbon-impregnated catheters. See text for summary and comments.

41 Falagas ME, Bliziotis IA, Fragoulis KN. Oral rifampin for eradication of

- Staphylococcus aureus carriage from healthy and sick populations: a systematic review of the evidence from comparative trials. Am J Infect Control 2007; 35:106-114.

A review of the effectiveness, safety and impact on the emergence of resistance of rifampicin-based regimens in eradicating $S$. aureus carriage. See text for summary and comments.

42 Darouiche RO, Raad II, Heard SO, et al. A comparison of two antimicrobial-impregnated central venous catheters. N Engl J Med 1999; 340:1-8.

43 Brun-Buisson C, Doyon F, Sollet JP, et al. Prevention of intravascular catheter-related infection with newer chlorhexidine-silver sulfadiazinecoated catheters: a randomized controlled trial. Intensive Care Med 2004; 30:837-843.

44 Rupp ME, Lisco SJ, Lipsett PA, et al. Effect of a second-generation venous catheter impregnated with chlorhexidine and silver sulfadiazine on central catheter-related infections: a randomized, controlled trial. Ann Intern Med 2005; 143:570-580.

45 Ostendorf T, Meinhold A, Harter C, et al. Chlorhexidine and silver-sulfadiazine coated central venous catheters in haematological patients - a double-blind, randomised, prospective, controlled trial. Support Care Cancer 2005; 13: 993-1000.

46 Gastmeier P, Geffers C. Prevention of catheter-related bloodstream infections: analysis of studies published between 2002 and 2005. J Hosp Infect 2006; 64:326-335.

47 Ranucci M, Isgro G, Giomarelli PP, et al. Impact of Oligon central venous catheters on catheter colonization and catheter-related bloodstream infection. Crit Care Med 2003; 31:52-59.

48 Stoiser B, Kofler J, Staudinger T, et al. Contamination of central venous catheters in immunocompromised patients: a comparison between two different types of central venous catheters. J Hosp Infect 2002; 50:202-206.

49 Corral L, Nolla-Salas M, lbanez-Nolla J, et al. A prospective, randomized study in critically ill patients using the Oligon Vantex catheter. J Hosp Infect 2003; 55:212-219.

50 Bong JJ, Kite P, Wilco MH, McMahon MJ. Prevention of catheter related bloodstream infection by silver iontophoretic central venous catheters: a randomised controlled trial. J Clin Pathol 2003; 56:731-735.

51 Moretti EW, Ofstead CL, Kristy RM, Wetzler HP. Impact of central venous catheter type and methods on catheter-related colonization and bacteraemia. J Hosp Infect 2005; 61:139-145.
52 Borschel DM, Chenoweth CE, Kaufman SR, et al. Are antiseptic-coated - central venous catheters effective in a real-world setting? Am J Infect Control 2006; 34:388-393.

A prospective assessment of the clinical and economic effectiveness of antiseptic-coated catheters for critically ill patients after their introduction for all patients requiring central venous access in the ICU. See text for summary and comments.

53 Safdar N, Maki DG. Use of vancomycin-containing lock or flush solutions for - prevention of bloodstream infection associated with central venous access devices: a meta-analysis of prospective, randomized trials. Clin Infect Dis 2006; 43:474-484.

A meta-analysis of the data on the efficacy of vancomycin-containing lock or flush solutions collected from seven randomized, controlled trials involving a total of 463 patients (five in ICUs). The use of these solutions reduced the risk of intravascular device-related infection. The authors concluded that the use of an anti-infective lock solution warrants consideration for patients who require central access but who are at high risk of bloodstream infections, such as patients with malignancy or low-birthweight neonates.

54 Van de Wetering MD, Van Woensel JBM. Prophylactic antibiotics for pre- venting early central venous catheter Gram positive infections in oncology patients. Cochrane Database Syst Rev 2007; CD003295.pub2

A meta-analysis of the data from nine trials with a total of 588 patients. The overall effect of administering an antibiotic before the insertion of the catheter did not significantly decrease the number of Gram-positive long-term tunnel'ed CVC infections. Flushing them with antibiotics and heparin significantly reduces the infection rate. The authors concluded that flushing the catheter with a vanco/ heparin lock solution had a positive overall effect.

55 Segarra-Newnham M, Martin-Cooper EM. Antibiotic lock technique: a review of the literature. Ann Pharmacother 2005; 39:311-318.

56 Pennington $\mathrm{CR}$, Pithie AD. Ethanol lock in the management of catheter occlusion. J Parenter Enter Nutr 1987; 11:507-508.

57 Kerner JA Jr, Garcia-Careaga MG, Fisher AA, Poole RL. Treatment of catheter occlusion in pediatric patients. J Parenter Enter Nutr 2006; 30 : S73-S81.

58 Dannenberg $\mathrm{C}$, Bierbach $\mathrm{U}$, Rothe A, et al. Ethanol-lock technique in the treatment of bloodstream infections in pediatric oncology patients with broviac catheter. J Pediatr Hematol Oncol 2003; 25:616-621.

59 Onland W, Shin CE, Fustar S, et al. Ethanol-lock technique for persistent

- bacteremia of long-term intravascular devices in pediatric patients. Arch Pediatr Adolesc Med 2006; 160:1049-1053.

A retrospective analysis of the 51 ethanol-lock treatments in 40 children. The authors concluded that their data support the use of the ethanol-lock technique in conjunction with systemic antibiotics as an effective and safe method to retain the use of previously infected central venous catheters.

60 Ackoundou-N'guessan C, Heng AE, Guenu S, et al. Ethanol lock solution as an - adjunct treatment for preventing recurrent catheter-related sepsis - first case report in dialysis setting. Nephrol Dial Transplant 2006; 21:3339-3340.

The first documented case report of the use of an ethanol lock technique as adjunctive treatment for preventing dialysis catheter infection in a patient with recurrent bacteraemia.

61 Crnich CJ, Halfmann JA, Crone WC, Maki DG. The effects of prolonged ethanol exposure on the mechanical properties of polyurethane and silicone catheters used for intravascular access. Infect Control Hosp Epidemiol 2005; 26:708-714. 